\title{
Urinary and synovial pyridinium crosslink concentrations in patients with rheumatoid arthritis and osteoarthritis
}

Luigi Sinigaglia, Massimo Varenna, Lucia Binelli, Francesco Bartucci, Marialisa Arrigoni, Roberto Ferrara, Gianalfredo Abbiati

\begin{abstract}
Objectives-To assess urinary and synovial concentrations of hydroxypyridinium crosslinks of collagen in patients with rheumatoid arthritis (RA) and osteoarthritis (OA) and to evaluate whether a combined measurement in the two compartments could give additional information about the origin of these compounds in joint diseases.
\end{abstract}

Methods-Concentrations of hydroxypyridinoline (HP) and lysylpyridinoline (LP) were measured by high pressure liquid chromatography in urinary and synovial samples collected from 20 patients with RA and 20 patients with knee OA. Full laboratory and clinical assessments were performed.

Results-Urinary concentrations of both $H P$ and LP were significantly greater in $R A$ than in OA. Urinary HP in RA correlated with the number of swollen joints corrected for Lansbury index and with erythrocyte sedimentation rate and $\mathrm{C}$ reactive protein. In synovial fluid from both groups, only relatively small amounts of HP were measured, while bone type I collagen specific LP was below the limit of detection in all samples. In RA patients, but not in OA patients, there was a strong correlation between urinary and synovial concentrations of HP $(r=0 \cdot 75)$.

Conclusions-The results underline the relationship between urinary $H P$ and disease extent and activity in RA. The findings in synovial fluid support the hypothesis of an extraskeletal origin of HP in chronic joint diseases in which cartilage and synovial turnover may be increased.

(Ann Rheum Dis 1995; 54: 144-147)

The pyridinium derivatives hydroxylysylpyridinoline (HP) and lysylpyridinoline (LP) are intermolecular crosslinks present in the mature form of collagen. During the process of collagen breakdown, the two crosslinks are released into blood and are subsequently excreted in urine. ${ }^{1}$ While LP is considered a specific marker of bone and dentine type I collagen degradation, HP is more widely distributed and has been shown to be retained in considerable amounts also in all three cartilage specific collagens: types II, IX and $\mathrm{XI}^{2}{ }^{2}$ Several studies reported that urinary excretion of pyridinium crosslinks provides a reliable clinical index of bone turnover in patients with metabolic bone disorders. ${ }^{34}$ Increased amounts of urinary pyridinolines in patients with joint diseases such as rheumatoid arthritis (RA) $)^{5-8}$ or osteoarthritis (OA) ${ }^{9-11}$ have been reported, but definitive information about their potential source is lacking. Furthermore, no data exist concerning their concentration in synovial fluid. The purpose of this study was to evaluate the relationship between urinary excretion of pyridinium crosslinks and clinical variables in arthritic diseases, and to measure crosslinks concentrations in the synovial compartment in order to test the hypothesis that a combined measurement could give additional information about the origin of these compounds in arthritic diseases.

\section{Patients and methods}

Samples of urine, serum and synovial fluid were collected from a total of 40 in- and outpatients attending the Department of Rheumatology at the University of Milan.

The first group of patients included 20 subjects (four male) of mean age $57 \cdot 7$ (SD 13.8) years, affected by RA diagnosed according to the 1987 criteria of the American Rheumatism Association (ARA), with a median duration of disease of 50.8 (range 11-240) months and synovial fluid effusion as a result of active synovitis of the knee. General exclusion criteria from this group were age $<20$ or $>75$, ARA functional Class IV, changes in pharmacotherapy within six months before the study, renal or hepatic impairment, clinically overt osteoporosis or bone fractures within one year before entering the study. Clinical examination included body mass index, number of swollen joints corrected for joint size according to Lansbury, ${ }^{12}$ duration of morning stiffness, ARA functional class. Acute phase reactants (erythrocyte sedimentation rate, $\mathrm{C}$ reactive protein), synovial white cell count, and cumulative and current steroid dosage were assessed for each patient. In this group, seven patients were receiving steroids with a mean daily dose of $2.57 \mathrm{mg}$ of 6-methyl prednisolone (range $2-6 \mathrm{mg}$ ).

The second group included 20 patients (six male) aged $61.8(8 \cdot 3)$ years affected by knee pain from gonarthrosis, selected by the criteria for $\mathrm{OA}$ as described by Lequesne. ${ }^{13}$ Prerequisites for entering the study were the 

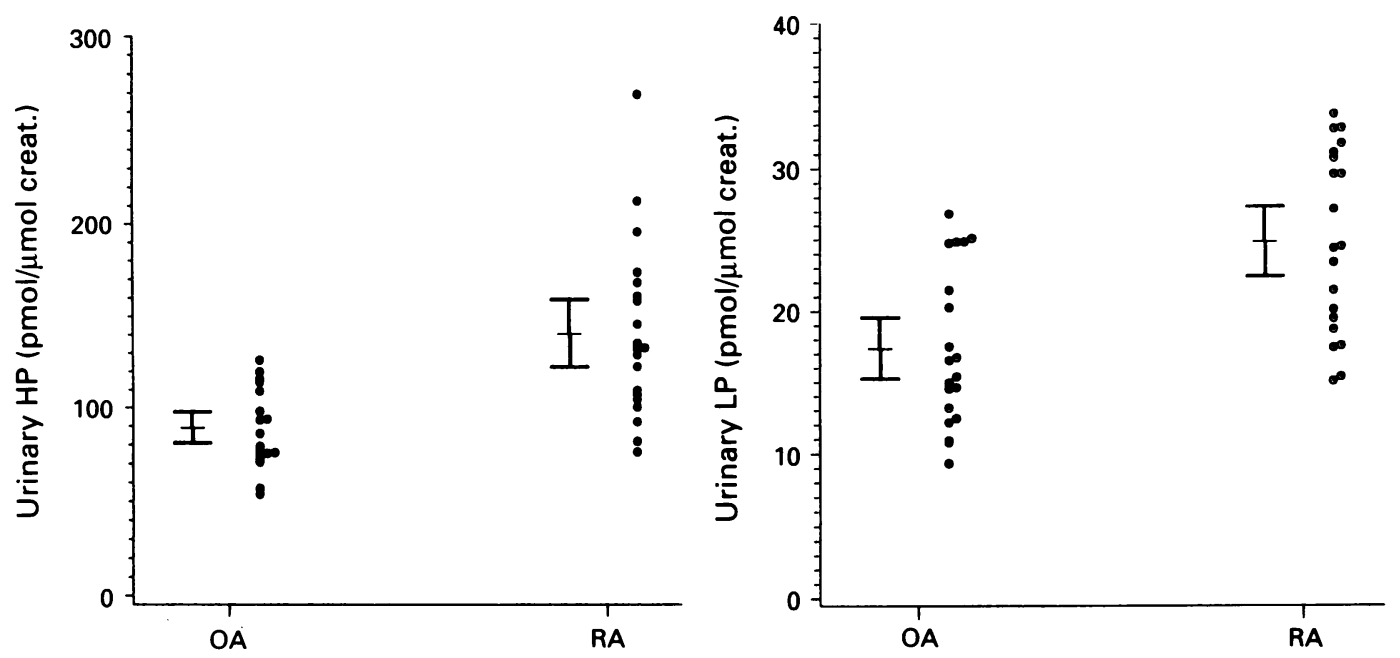

Figure 1 Raw values, mean and $95 \%$ confidence intervals of urinary concentrations of hydroxypyridinoline (HP) (left) and lysylpyridinoline (LP) (right) in patients with osteoarthritis $(O A)$ and rheumatoid arthritis $(R A)$.

presence of synovial effusion and a classification of grade 2 or less using the standard radiographs of Kellgren and Lawrence. ${ }^{14}$ Patients older than 75 were excluded, as were patients with osteoarthritis of other large joints and subjects with a history of osteoporotic fractures. Clinical and laboratory data assessed for each patient included body mass index, pain at rest and on walking graded by visual analogue scale, current medication, acute phase reactants, and synovial white cell count.

In each subject participating in the study, two hours fasting early morning urine samples and synovial samples were collected and analysed by high pressure liquid chromatography for pyridinium crosslinks according to the method of Abbiati et al $;^{15}$ the same method with minor modifications was used for synovial fluid determinations. Concentrations of HP and LP are expressed as pmol/ $\mu \mathrm{mol}$ of creatinine in urinary samples and $\mathrm{pmol} / \mathrm{ml}$ in synovial fluid samples.

Statistical analysis included Student's $t$ test and Pearson correlation coefficient.

\section{Results}

URINARY CONCENTRATIONS

Figure 1 shows the mean values and 95\% confidence intervals of urinary HP and LP in the two groups. The correlation between urinary concentrations of HP and LP was significant in both groups, but at a greater value in OA $(r=0.87)$ than in RA $(r=0.49)$. Mean urinary concentrations and $95 \%$ confidence intervals of HP were $139 \cdot 1$ (117 to $161.3) \mathrm{pmol} / \mu \mathrm{mol}$ in RA and $87.6(77.5$ to $97.7) \mathrm{pmol} / \mu \mathrm{mol}$ in OA $(\mathrm{p}=0.0001)$; those of LP were $24.6(21.6$ to $27 \cdot 6) \mathrm{pmol} / \mu \mathrm{mol}$ in RA and $17 \cdot 1(14.5$ to $19 \cdot 7) \mathrm{pmol} / \mu \mathrm{mol}$ in OA $(\mathrm{p}=0.002$ ) (fig 1). In RA patients, data revealed a lack of correlation between urinary concentrations of LP and disease activity indexes, while a quite good correlation was detected between urinary concentrations of HP and both the number of swollen joints corrected for joint size according to Lansbury, and acute phase reactants (table). In RA, urinary concentrations of crosslinks were similar in those receiving and not receiving steroids, and did not correlate with cumulative or actual corticosteroid dose, duration of disease, morning stiffness, and ARA functional class. In OA patients, no correlation was found with grading of pain at rest and on walking and with current use of non-steroidal antiinflammatory drugs.

SYNOVIAL CONCENTRATIONS

In the synovial fluid of both $\mathrm{RA}$ and $\mathrm{OA}$ patients, relatively large amounts of HP were measured (RA mean 25.3 (SD 13.5) pmol $/ \mathrm{ml}$; OA mean $23.2(8.4) \mathrm{pmol} / \mathrm{ml})$, whereas the concentrations on type I collagen specific LP were below the limit of detection $(<1 \mathrm{pmol} / \mathrm{ml})$ in all samples from both groups. We found a strong correlation between urinary and synovial concentrations of HP $(r=0.75 ; p<0.0001)$ (fig 2) in RA patients but not in OA patients. In steroid treated RA patients, synovial concentrations of HP were significantly smaller than in subjects who were not receiving steroids (mean $16 \cdot 1$ (SD $7 \cdot 7$ ) $\mathrm{pmol} / \mathrm{ml} v$ $28.7(12.9) \mathrm{pmol} / \mathrm{ml}$, respectively; $\mathrm{p}=0.03)$. No correlation between synovial concentrations of HP and white cell count in synovial fluid was detected in either group.

\section{Discussion}

Our data confirm preliminary results showing that the rate of urinary excretion of pyridinium crosslinks is increased in patients with joint diseases. In each group studied, greater concentrations were found than in the control group of Seibel et $a l^{5}$ which included subjects

Correlation coefficients of urinary crosslinks with disease parameters in 20 patients with rheumatoid arthritis

\begin{tabular}{lll}
\hline & $H P$ & $L P$ \\
\hline Erythrocyte sedimentation rate & $0 \cdot 57^{\star}$ & $0 \cdot 08$ \\
C reactive protein & $0 \cdot 58^{\star}$ & $0 \cdot 07$ \\
Lansbury index & $0 \cdot 62^{\star}$ & $0 \cdot 33$ \\
No of swollen joints & $0 \cdot 45^{\star}$ & $0 \cdot 40$ \\
Disease duration & $-0 \cdot 25^{-}$ & $0 \cdot 26$ \\
HP & - & $0 \cdot 49^{\star}$
\end{tabular}

HP = Hydroxypyridinoline LP = lysylpyridinoline. ${ }_{\star_{p}}<0.05$. 


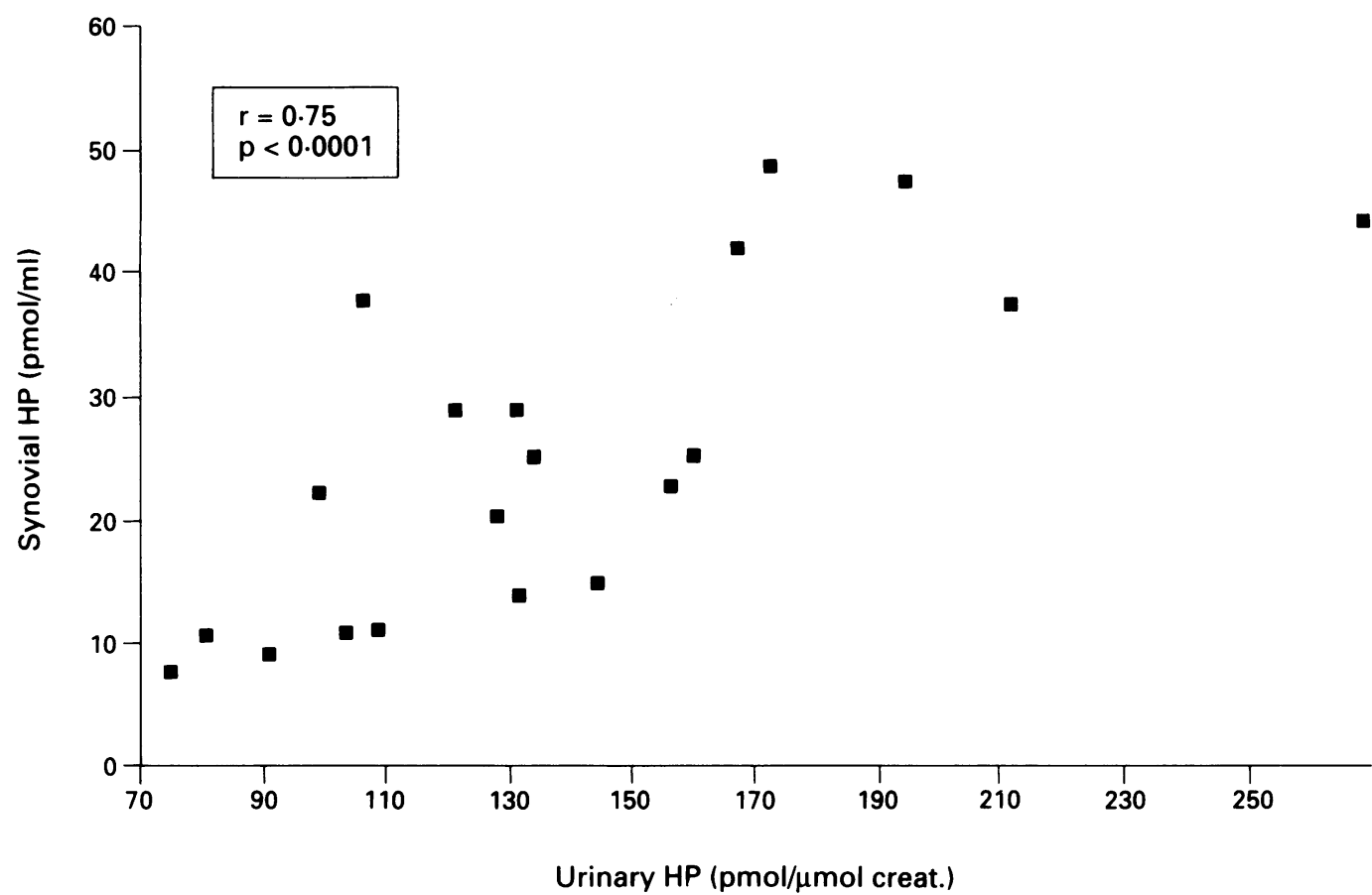

Figure 2 Correlation between urinary and synovial concentrations of hydroxypyridinoline (HP) in 20 patients with rheumatoid arthritis.

with no clinical evidence of joint damage. The urinary concentrations of both crosslinks in RA patients were significantly greater than in patients with gonarthrosis. This could partly reflect the increased systemic or local bone turnover in RA, in which excretion of pyridinium crosslinks has recently been found to correlate with the appendicular bone loss occurring in the early stage of the disease. ${ }^{8}$ Nevertheless, our results confirm preliminary data showing a close correlation between urinary excretion of $\mathrm{HP}$ and indices of disease activity in RA. ${ }^{6}$ Interestingly, in our study the best correlation was found with an articular index closely reflecting disease extent, such as the Lansbury index. In contrast to others, ${ }^{9-11}$ in OA patients we did not find any correlation with clinical indices, but our findings in $\mathrm{OA}$ were limited to a uniform sample of early gonarthrosis, with a relatively mild radiological score. It must be pointed out that differences in urinary concentrations of crosslinks between OA patients and normal subjects must be considered with caution because, in age matched controls, subclinical joint or spine disorders cannot be easily excluded and this may represent a bias when comparing patients with osteoarthritis with 'healthy' subjects.

The major finding in this study was the presence of HP, but not of LP, in synovial fluids of both RA and OA patients. This implies that in this compartment there is a sharp change in the ratio between the two crosslinks which can be estimated to be at least $20: 1$. This finding strongly supports the hypothesis that synovial HP in joint diseases depends on the degradation of an extraosseous source of collagen, reflecting a catabolic pathway other than skeletal or subchondral bone turnover. The highly significant correlation between synovial and urinary HP in RA could support the possibility that synovial HP may come, at least partially, from the circulation, but if that were so, relatively greater concentrations of LP would have been expected to be found in the synovial fluids. It is more likely that HP in synovial fluid of $\mathrm{RA}$ and $\mathrm{OA}$ patients derives from the breakdown of collagen which takes place in cartilage or in synovial tissue, as HP is the major crosslink in cartilage collagens, and articular cartilage has the highest concentration in any tissue of the body. ${ }^{5}$ Other authors have also reported significant amounts of $\mathrm{HP}$ in synovial tissue from patients with RA. ${ }^{5}$ Our data do not allow any conclusion as to the relative validity of the two hypotheses, but the finding of quantitatively similar synovial fluid concentrations of HP in two conditions with a highly different metabolic turnover of synovial membrane supports the possibility that synovial fluid HP can be at least in part derived from the degradation of the articular cartilage matrix. In contrast, the lack of correlation between urinary and synovial concentrations of $\mathrm{HP}$ in the OA group which exhibited synovial concentrations of HP similar to those in RA can be explained by an adjunctive fraction of synovium derived HP in the fluids of RA patients. This could also explain the greater concentrations of urinary crosslinks found in RA and the close correlation between HP and disease extent and activity in our patients affected by a polyarticular disease such as RA.

In conclusion, our observations suggest that increased urinary HP in joint diseases can be attributed at least in part to the breakdown of an extraskeletal source of collagen and may represent an index of overall joint involvement in RA. This conclusion is supported by the findings in synovial fluid, but our data do not allow a conclusion about a cartilaginous rather than synovial derivation of HP. In vitro and in vivo experimental studies must be planned 
to define the origin of crosslinks in joint diseases and to evaluate correlations with different stages of joint damage. Further prospective studies are also necessary to establish whether urinary or synovial pyridinolines alter in response to drug therapy, thus providing information for monitoring the progression of joint diseases.

1 Gunja-Smith Z, Boucek R Y. Collagen crosslink components in human urine. Biochem $\mathcal{f} 1981 ; 197$ 759-62.

2 Eyre D R, Wu J J, Woods P E, Weis M A. The cartilage collagens and joint degeneration. Brf Rheumatol 1991; 30 (suppl 1): $10-5$.

3 Uebelhart D, Gineyts E, Chapuy M C, Delmas P. Urinary excretion of pyridinium crosslinks: a new marker of bone resorption in metabolic bone disease. Bone Miner 1990, 8: 87-96.

4 Robins S P, Black D, Paterson C R, Reid D M, Duncan A, Seibel M J. Evaluation of urinary hydroxypiridinium crosslinks measurements as resorption markers in metabolic bone diseases. Eur 7 Clin Invest 1991; 21: 310-5.

5 Seibel M J, Duncan A, Robins S P. Urinary hydroxypyridinium crosslinks provide indices of cartilage and 16: $964-70$.

6 Black D, Marabani M, Sturrock R D, Robins S P. Urinary excretion of hydroxypyridinium crosslinks of collagen in patients with rheumatoid arthritis. Ann Rheum Dis 1989 48: $641-4$.
7 Spector T D, James I T, Hall G $\mathrm{M}$, Thompson $\mathbf{P}$ W, Perrett D, Hart D J. Increased levels of urinary collagen crosslinks in females with rheumatoid arthritis. Clin crosslinks in females with

8 Gough A K S, Peel N F A, Eastell R, Holder R L, Lilley J, Emery P. Excretion of pyridinium crosslinks correlates with disease activity and appendicular bone loss in early rheumatoid arthritis. Ann Rheum Dis 1994; 53: 14-7.

9 MacDonald A G, McHenry P, Robins S P, Reid D M. Relationship of urinary pyridinium crosslinks to disease extent and activity in osteoarthritis. Br f Rheumatol 1994; 33: 16-9.

10 Thompson P W, Spector T D, James I T, Henderson E Hart D J. Urinary collagen crosslinks reflect radiographic severity of knee osteoarthritis. Br ₹ Rheumatol 1992; 31: 759-61.

11 Astbury C, Bird H A, McLaren A M, Robins S P. Urinary excretion of pyridinium crosslinks of collagen correlated with joint damage in arthritis. $B r \mathcal{F}$ Rheumatol 1994; 33 $11-5$.

12 Lansbury J, Baier H N, McCraken S. Statistical study of variation in systemic and articular indexes. Arthritis Rheum 1962; 5: 445-57.

13 Lequesne $M$. Clinical features, diagnostic criteria, functional assessment and radiological classification of osteoarthritis (excluding the spine). Rheumatology 1982; 7: $1-10$.

14 Kellgren J H, Lawrence J S. Radiological assessment of osteoarthritis. Ann Rheum Dis 1957; 16: 494.

15 Abbiati G, Rigoldi M, Mussini E, Castiglioni C. An isocratic method for pyridinoline (HP) and deoxypyridinoline (LP) method for pyridinoline (HP) and deoxypyridinoline (LP) analysis; a new approach to their standard quantification. In: Christiansen $\mathrm{C}$, Overgaard $\mathrm{K}$, eds. Proceedings of the 3rd International Symposium on Osteoporosis, Copenhagen, 1990: 616-8. 